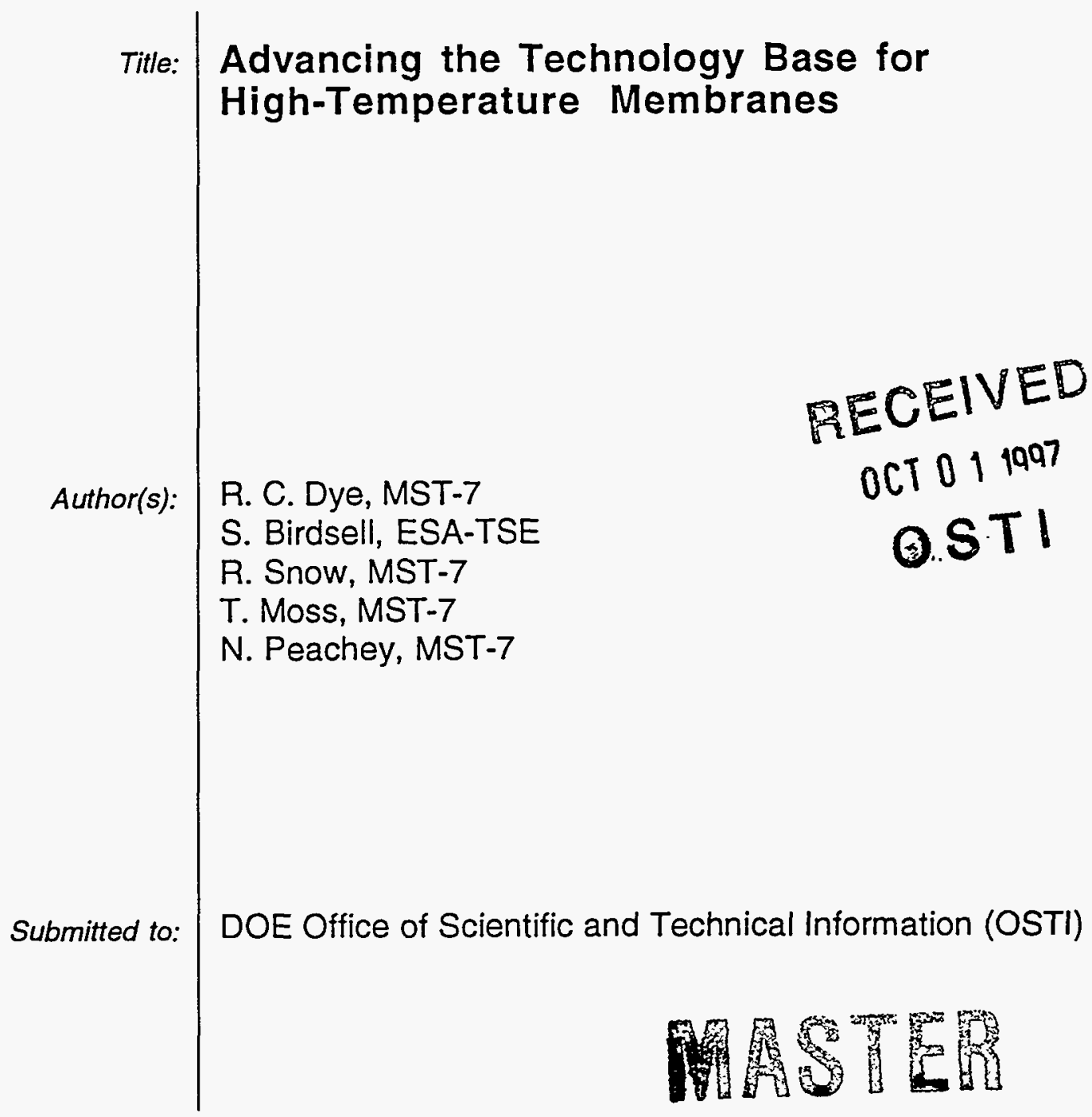

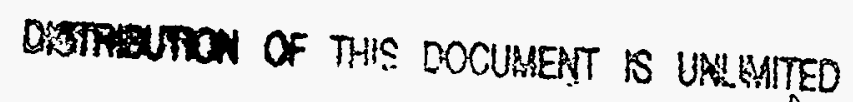

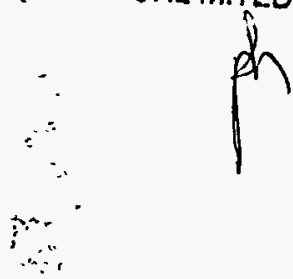

Los Alamos National Laboratory, an aftirmative action/equal opportunity employer, is operated by the University of Calfornia for the U.S. Department of Energy under contract W-7405-ENG-36. By acceptance of this article, the publistier recognizes that the U.S. Government retains a nonexclusive, royaltyfree license to publish or reproduce the published form of this contribution, or to allow others to do so, for U.S. Government purposes. Los Alamos Natlonal Laboratory requests that the publisher identify this article as work performed under the auspices of the U.S. Department of Energy. Los Alamos National Laboratory strongly supports academic freedom and a researcher's right to publish; as an institution, however, the Laboratory does nol endorse the viewpoint of a publication or guarantee its technical correctness. 


\section{DISClaAMER}

Portions of this document may be illegible in electronic image products. Images are produced from the best available original document. 


\section{DISCLAIMER}

This report was prepared as an account of work sponsored by an agency of the United States Government. Neither the United States Government nor any agency thereof, nor any of their employees, make any warranty, express or implied, or assumes any legal liability or responsibility for the accuracy, completeness, or usefulness of any information, apparatus, product, or process disclosed, or represents that its use would not infringe privately owned rights. Reference herein to any specific commercial product, process, or service by trade name, trademark, manufacturer, or otherwise does not necessarily constitute or imply its endorsement, recommendation, or favoring by the United States Government or any agency thereof. The views and opinions of authors expressed herein do not necessarily state or reflect those of the United States Government or any agency thereof. 


\title{
Advancing the Technology Base for High-Temperature Membranes
}

\author{
Robert C. Dye*, Stephen A. Birdsell, Ronny C. Snow, \\ Thomas S. Moss, and Nathaniel Peachey
}

\begin{abstract}
This is the final report of a two-year, Laboratory Directed Research and Development (LDRD) project at the Los Alamos National Laboratory (LANL). This project addresses the major issues confronting the implementation of high-temperature membranes for separations and catalysis. We are pursuing high-temperature membrane systems that can have a large impact for DOE and be industrially relevant. A major obstacle for increased use of membranes is that most applications require the membrane material to withstand temperatures above those acceptable for polymer-based systems. Advances made by this project have helped industry and DOE move toward high-temperature membrane applications to improve overall energy efficiency.
\end{abstract}

\section{Background and Research Objectives}

Although the development of high-temperature membranes is still in its infancy, it is a technology which, when fully mature, will yield immense returns in the chemical processing industry, energy conservation, as well as the fuel cell technology. The 1991 Report of the National Critical Technologies Panel identified energy and environment as one of the six major critical areas of interest. Membranes will make possible the achievement of significant gains in both energy conservation and waste management and remediation. With disposal costs reaching in excess of $\$ 240$ per ton as landfill space is being depleted, the implementation of separation technologies including membranes for waste recycling, treatment, and remediation are becoming increasingly more urgent. Intimately connected to energy conservation is the improvement in efficiency of chemical processes. Improved catalysis derived from the ability of membranes to simultaneously serve as catalysts and separation devices could help to dramatically reduce energy consumption while also improving productivity. The worldwide market for catalysts in 1993 was $\$ 7.8$ billion and the petrochemical industry, for which catalysts are particularly important, currently accounts for $\$ 400$ billion in sales and 1.8 million jobs. Inorganic membranes have distinct advantages over existing polymer and organic membranes in that

"Principal Investigator, email: rcdye@lanl.gov 
they can withstand the high temperatures associated with many chemical processing procedures, are well suited to chemically harsh environments, and often provide better gas flows. Since many potential petrochemical applications include one or more of these conditions, inorganic membranes are essential in realizing improvements necessary to sustain technological development.

In spite of the overwhelming potential for high-temperature, inorganic membranes, much remains to be done before their capabilities are fully developed. Although the field is experiencing a healthy $15 \%$ growth per year, the combined sales for 1993 were a mere $\$ 40$ million. To aid in this development, we have launched an effort in investigation of inorganic membranes. This involves both the membrane formation using a variety of deposition techniques and the testing of their characteristics. A high-temperature testing apparatus has been designed and built which has the capability of investigating properties such as permeabilities and diffusion coefficients between room temperature and $1200^{\circ} \mathrm{C}$. Several thin films have been deposited for investigation as membranes. Deposition techniques include pulsed laser deposition (PLD), chemical vapor deposition (CVD), and physical vapor deposition (PVD). A zeolitic membrane which has been deposited using PLD has been tested and has shown a hydrogen/argon separation ratio of 5.3. Since this is higher than that expected for Knudsen flow (4.45), it suggests that this microporous membrane is capable of molecular sieving. This membrane is of interest not only for gas separations applications, but since it contains zeolites, it could be incorporated into a catalytic membrane reactor.

Also, the use of hydrogen gas has become more important in recent years to a variety of high technology areas. The steady depletion of limited-resource fossil fuels, such as light crudes and natural gas, and the associated pollution problems have made hydrogen based energy systems more attractive. In the microelectronics industry, there is a growing need for ultra-high purity gases as the line resolution continues to shrink and impurity tolerance levels become more stringent. Further, ultra-high purity hydrogen gas is needed for ferrous and nonferrous metals processing, chemical and polymer synthesis, and petrochemical processing. These applications, as well as others requiring hydrogen recovery and separation, have created and sustained an interest in hydrogen separation techniques.

A novel multilayer metal membrane has been developed that can be used for the separation of ultra-high purity hydrogen from impure feed streams. The membrane is comprised of very thin layers of dense palladium film deposited on both sides of a thin Group V metal foil, ion-milled prior to sputtering of the palladium. This membrane operates at elevated temperatures on the order of $300^{\circ} \mathrm{C}$ and is capable of high rates of 
hydrogen flow. Flows are dependent on the pressure differential applied to the membrane, but flows of 25 standard cubic centimeters of hydrogen per minute per square centimeter of membrane and higher are regularly observed with modest differentials. Testing of the membrane for a period of 775 hours showed stable flows under constant conditions. A membrane system has been successfully applied to a proton-exchange-membrane fuel cell and was tested using a pseudo-reformate feed stream containing $1 \%$ carbon monoxide without any degradation in performance.

\section{Importance to LANL's Science and Technology Base and National R\&D Needs}

This development of membranes is furthering hydrogen isotope separation and has several advantages over current cryogenic separation methods. First, the cost of such separations would be reduced since no cryogenic steps are necessary for the process and the composite metal membranes can be produced in larger areas at minimal cost. More important is the fact that membrane separation would avoid the high radiation resulting from the liquid tritium produced in the cryogenic process because the tritium would remain a gas throughout the separation. This would assist in keeping radiation exposure as low as reasonably achievable (ALARA). It would also minimize the risk of contamination by highly concentrated radiation sources, making accidental leakage a much less troublesome issue.

These membranes may also be utilized in the accelerator production of tritium for the recovery and extraction step. Also, the outlet streams of fusion reactors contain significant amounts of tritiated methane and water. The tritium should be removed from methane and water and recycled to the reactor because this species cannot be released to the environment and would be expensive to dispose of. Our approach is to convert the tritiated methane and water into hydrogen and carbon oxides using a membrane reactor, which will allow us to recover $99.999 \%$ of the tritium. This large conversion is possible due to the shift in equilibrium caused by hydrogen separation through the membrane. By using a multi-metal membrane in the membrane reactor the process will become less expensive and more efficient. The nuclear weapons program can use this approach to recover the tritium during dismantling.

A systematic study of the processes involved in membranes will allow the rational design of improved separation devices. This, along with modeling of the systems, will allow the determination of the constituents that limit the overall permeability of a particular membrane. Under the anticipated range of processing conditions and in these types of 
metal hydride systems, transport of hydrogen and its isotopes is usually quantum mechanical in nature. By examining the permeability of hydrogen and deuterium for the membranes, we will be able to tell whether or not the system possesses a reverse isotope effect. If this occurs the deuterium would move through the membrane faster than the hydrogen. Depending on the outcome of this investigation, one of several appropriate quantum models of permeability can be used to fit the data. The models are based on quantum corrections to transport behavior (e.g. diffusion), originating from Wigner, due to zero point motion and tunneling phenomena. These fits can then be used to predict the behavior of other membranes and of tritiated systems. A well-developed grasp of these issues would then permit a more rational approach to membrane modification and design leading to increased efficiency of separations. The next step is to take the model and experimental results from the deuterium and apply them to the separation of tritium. In the short term, over 2.5 trillion standard cubic feet of hydrogen is wasted each year by U.S. refineries because it is more cost effective to burn than separate from waste streams. Clearly, an improved membrane recovery system could save a large portion of this wasted fuel. Our research can enhance the present state of hydrogen technologies by making hydrogen separation more cost effective.

Improved membranes for gas separation also have major ramifications for fuel cell systems. DOE estimates that 2-4 million barrels of oil per day could be saved and an economic benefit of $\$ 42$ billion per year (based on fuel savings) could be realized by the year 2030 by using methanol powered fuel cell vehicles. Successful development of membranes for membrane reactors could increase these savings in two ways. First, methanol for use in fuel cell vehicles could be produced more efficiently from coal or natural gas than by conventional processing methods. Similarly, hydrogen production from methanol onboard the vehicles could be accomplished more efficiently. If the overall efficiency of both conversions could be increased by a total of $20 \%$ using membrane reactors, then the economic benefit would increase by an additional $\$ 5$ billion per year.

\section{Scientific Approach and Accomplishments}

To exploit the rapid bulk diffusion of hydrogen in the refractory metals, a composite structure has been fabricated where palladium is placed on each side of the refractory metal chosen for its ability to transport hydrogen and to offer structural integrity for the composite membrane. This construction allows the dissociation of the molecular hydrogen into atomic hydrogen by the palladium surface layer, rapid transport of the atomic 
hydrogen through the refractory metal, and reassociation into molecular hydrogen on the opposite palladium surface. Such a structure has several advantages. First, greater overall hydrogen fluxes are possible because the diffusion is not limited by the fcc structure of the palladium. Because of this, the membrane can be thicker providing improved mechanical/structural properties while still providing acceptable, and even improved, gas fluxes. Second, since the refractory metals are significantly less expensive than palladium, these membranes are much more economical because only two thin layers of palladium are needed. Further, while the Group V metals are subject to hydrogen embrittlement, this regime is only a problem well below room temperature. Should the palladium layer develop defects, the membrane would still be functional because the defect would only expose a small area of the refractory metal. This composite membrane structure is illustrated in Figure 1.

These advantageous properties have been employed by other groups and a number of patent applications have been filed and granted over recent years. However, these groups did not address the problems of surface oxides and contamination on the refractory metals. We recognized that the hydrogen fluxes could be improved by eliminating the boundary layers to obtain a highly clean surface on the refractory metal and by depositing an exceptionally pure palladium layer with particular crystallographic orientations. A patent application has been filed on this process and material, and a full description of the initial work has been given elsewhere (see Publications). This report describes the advances in the membrane performance and understanding since that point.

The fabrication of the composite membrane was done according to a set procedure where the foil was first cleaned with soap and water followed by a solvent rinse to remove any surface oils. The foil was then mounted into a vacuum chamber which was then pumped down to the range of $10^{-6}$ torr. The foil was then cleaned using an argon ion gun to remove the native surface oxide layer. Without breaking the vacuum, layers of palladium of various thicknesses were sputtered onto the front and back of the foil. Deposition thickness was monitored using a crystal monochromater, and the foil was kept at ambient temperature during cleaning and deposition. The membrane was later removed from the vacuum chamber and tested for hydrogen permeability. The composite membrane was fully dense with no residual porosity in either the palladium coating or the metal foil.

The membrane test system consisted of a membrane holder unit in which the membrane was sealed between the feed and permeate streams. The feed flows were composed of reagent grade hydrogen and ultra-high purity argon and were controlled using independent MKS mass flow controllers so that mixtures of various compositions could be used. The pressure on the feed side of the membrane was controlled by either pumping 
using a turbo pump (in the case of subatmospheric pressures) or restricting the flow of the exhaust from the feed side using a needle valve (in the case of elevated pressures). The pressure of the feed was measured using a Baritron pressure transducer for pressures up to 1000 torr and using a dial guage for higher pressures. The permeate side of the membrane allowed the gas flow to pass through an MKS mass flow meter to determine the flow rate of gas passing through the membrane. The pressure on the permeate side of the membrane was also controlled either by pumping for low pressures or by restricting the flow for high pressures. The composition of both the feed and permeate gas streams could be analyzed using a Residual Gas Analyzer (RGA). Leaks could be detected by the significant presence of argon in the permeate gas stream, indicating that argon was leaking through or around the seal of the membrane.

One of the first questions that must be answered regarding the fundamental theory behind the composite membrane is: Does it only allow hydrogen to pass through? To demonstrate this, an experiment was set up where a feed gas mixture of equal parts of hydrogen, helium, and argon was introduced into the system. The permeate gas stream was monitored for composition using the RGA. The helium signal is present at intensities over four orders of magnitude below that of the hydrogen signal. No argon signal was discernible from the background. Based on this data, the permeate stream would be approximately $99.998 \%$ hydrogen, with the balance helium; this value corresponds to a hydrogen:helium selectivity of 49,999:1. However, it is worth mentioning that the helium signal is in the lower range of detection of the RGA, and the certainty of its value is not as high as that of the hydrogen. As a result, the actual hydrogen purity may be higher than was calculated from the raw numbers.

This experiment demonstrates that the membrane is able to produce extremely high hydrogen gas purities. Most importantly, it also shows that the method of purification is solid-state diffusion of hydrogen through the membrane, as was described previously, rather than by transport through pores. Because hydrogen atoms have an effective size that is larger than helium atoms, i.e., $2.89 \AA$ for $\mathrm{H}_{2}$ versus $2.6 \AA$ for He, a higher flow rate of helium would be expected if the method of transport was through pores. Any flow through the membrane must then be explained as the interaction of the hydrogen gas with the membrane in such a way that only allows hydrogen to pass through the membrane. The slight presence of helium is not totally unexpected because the palladium film and vanadium or tantalum foil were polycrystalline. Thus, some flow of helium could take place along the grain boundaries, especially with its small size. For much the same reason, any flaws in the seal around the membrane might allow a small amount of helium to leak through. Most of all, the high hydrogen/helium selectivity from an even gas mixture shows that the 
membrane is capable of producing ultra-high purity hydrogen flows and functions as theorized as a solid-state transport mechanism.

If the properties have been properly engineered, the composite membrane must also show increased performance to that seen by pure palladium. This was tested by comparing a multilayer metal membrane to a sheet of palladium foil with comparable thickness. Both membranes were tested at identical temperatures, pressures, and flow rates to produce a fair comparison. It is possible to see that the use of the composite membrane provides superior performance in terms of flow rate compared to that of pure palladium. Once steady state is reached, the composite membrane showed a factor of ten improvement in the flow rate over the pure palladium membrane. This increase shows that it is indeed possible to increase the hydrogen flow by replacing the bulk palladium with a material that has much higher transport rates.

The long-term performance of the membrane is another question that must be properly addressed, because the membrane must be able to withstand long periods of continuous operation without any degradation. An example of the flow rate in standard cubic centimeters per minute ( $\mathrm{sccm}$ ) as a function of time in a 575-hour test is shown in Figure 2; this experiment was run at a membrane temperature of $300^{\circ} \mathrm{C}$, feed pressure of 600 torr (ambient pressure in Los Alamos, NM), permeate pressure of 17 torr, a hydrogen flow rate of $100 \mathrm{sccm}$ and an Ar flow rate of $35 \mathrm{sccm}$. The experiment was stopped due to the emptying of a hydrogen D-cylinder and could have conceivably continued for many more hours. This membrane was tested for an additional 200 hours under a variety of flow rates and feed pressures prior to the 575-hour test, making the total testing time 775 hours. The flow rate per area of membrane was stable at $7.78 \mathrm{sccm}$ per $\mathrm{cm}^{2}$ which is a low flow rate for this membrane system. However, that value corresponded to an $84 \%$ efficiency, i.e., $84 \%$ of the hydrogen in the feed stream was transported across the membrane.

The flow rate can be seen to drop from an initial value and approach a steady state value. This drop in the flow rate is reproducible within the membrane. This initial drop in the flow rate is thought to be caused by the release of the feed gas flow from the main control valve. The pressure upstream of the valve can approach the bottle pressure values of 2 to $2.5 \mathrm{~atm}$ and when this pressure is released, it creates an abnormally high flux of gas to the membrane surface. However, over the next few minutes, the pressure and flow rates approach their steady state values, and the observed flux through the membrane approaches steady state.

Variation of the flow rate with membrane temperature also shows a positive relationship. As a test of this, a membrane was used at six different temperatures from 275 to $375^{\circ} \mathrm{C}$ under identical flow and pressure conditions. A value of the steady state flow rate 
through the membrane was then determined after allowing the membrane flow to equilibrate. We found that the flow rate increases with the temperature, and the relationship appears to be almost linear within the temperature range explored. However, the increase in flow rate does not mean that operating the membrane at the highest possible temperature is advisable because the diffusion between the coating and the foil will also increase exponentially with the membrane temperature, especially with a flow of hydrogen passing through the membrane. Any diffusion between the two materials risks degradation of the flow rate because it will cause a high energy barrier to the diffusion of hydrogen atoms through the membrane. The diffusion also risks the formation of brittle intermetallic phase(s) that might provide a fracture point under high pressure-differential conditions.

An optimum operating temperature of $300^{\circ} \mathrm{C}$ has been determined; this temperature provides a high flow rate of hydrogen while limiting any diffusion between the palladium and foil. A foil that was run under steady state conditions for 100 hours at $300^{\circ} \mathrm{C}$ and a one atmosphere pressure differential was examined using Rutherford Backscatter Spectroscopy. The results of this characterization showed that the membrane had experienced no interdiffusion of palladium into the vanadium foil. In fact the two spectra of the membrane, one that had not been used and another that had 100 hours of use, could be laid one on top of another without being able to distinguish between the two, indicating a clean interface between the two materials.

Another area of interest is the determination of the limiting step within the membrane for hydrogen transport. The overall transport process can be broken into three steps that must occur at or in the membrane. The first step is the molecular hydrogen adsorption onto the palladium surface and dissociation into atomic hydrogen. The next step is the atomic hydrogen diffusion into, through, and out of the bulk metals. Finally, the atomic hydrogen re-associates into molecular hydrogen on the downstream palladium layer and desorbs from the surface. If the transport process is limited by either step one or three (they are the same process in forward and reverse), then the flow rate of hydrogen through the membrane is a linear function of the hydrogen pressure differential between the feed and permeate sides. If the bulk diffusion of the atomic hydrogen limits the flow rate, the transport rate becomes governed by Sievert's law and is a linear function of:the differential of the square root of the hydrogen partial pressure. Based on a series of experimental conditions, it was determined that the flow rate was best fit by using the square root of the differential pressure. If this is indeed the case, then it is anticipated that the flow rates can be increased by decreasing the thickness of the limiting metal, e.g., either palladium or the Group V. Because the hydrogen permeability of palladium is several orders of magnitude lower than that of tantalum or vanadium, it is expected that the diffusion of the atomic 
hydrogen through the palladium is limiting the hydrogen transport rate. Thus, thinner palladium should yield higher flow rates, as well as increased cost savings because of a reduction in the amount of material used and in the time needed for deposition.

The membrane may be applied to a number of different areas and technologies that require ultra-high purity hydrogen or high efficiency hydrogen recovery. Among these applications are semiconductor processing, commercial gas purification, metals processing, chemical and polymer synthesis, exhaust stream recovery, and environmental remediation. However, a technology of particular interest is that of proton-exchange-membrane fuel cells (PEMFC). In the PEMFC, hydrogen and oxygen are reacted to produce water and electricity from the electrochemical potential of the reaction. The PEMFC is capable of high power density, high chemical energy to electrical energy conversion, and fast and easy start-up. However, a major drawback is that the cathode of the fuel cell is easily poisoned by the presence of carbon monoxide in the range of parts per million (ppm) within the hydrogen stream. As a result, either an ultra-high purity hydrogen supply is needed or a lower purity gas supply must pass through shift and partial oxidation (PROX) reactors to effectively oxidize the carbon monoxide to carbon dioxide. The use of a membrane separation system could replace the need for the shift and PROX reactors because the membrane would only allow hydrogen to pass and would exclude any carbon monoxide from the cathode.

A PEMFC was operated at LANL where a pseudo-reformate gas mixture was introduced into a separation system before entering into the fuel cell. The pseudo-reformate gas supply was simulated for a methanol reformate feed and composed of $1 \% \mathrm{CO}, 24 \%$ $\mathrm{CO}_{2}$, and $74 \% \mathrm{H}_{2}$. The membrane system was operated at an external temperature of $315^{\circ} \mathrm{C}$ and the PEMFC was a $5 \mathrm{~cm}^{2}$ single cell. The performance of the fuel cell showed a current density of $600 \mathrm{~mA} / \mathrm{cm}^{2}$ at $0.6 \mathrm{~V}$. In contrast, the presence of only $100 \mathrm{ppm} \mathrm{CO}$ in the hydrogen fed directly into the anode suppressed the current density at this voltage to about $60 \mathrm{~mA} / \mathrm{cm}^{2}$, one tenth the cell performance. It is anticipated that the membrane separator will be equally effective with the much higher $\mathrm{CO}$ levels expected from natural gas reformers, as well as from other fuel sources. Also, this application shows that the membranes have very high selectivity between hydrogen and carbon monoxide, as can be further evidenced by the degradation in the performance of the fuel cell at even $20 \mathrm{ppm} \mathrm{CO}$.

The uses for this membrane center around areas that require ultra-high purity hydrogen or need hydrogen recovery from an impure gas stream. A membrane system has been successfully applied to a proton exchange membrane fuel cell, where it would replace the shift and PROX reactors that are needed to remove carbon monoxide from the hydrogen supply. The membrane system was tested using a pseudo-reformate (methanol) feed 
stream containing $1 \% \mathrm{CO}$ without any degradation in the fuel cell performance. When the same fuel cell was run with as little as 20 parts per million carbon monoxide, the fuel cell showed a serious reduction in performance.

\section{Publications}

1. Peachey, N.M., Snow, R. C., and Dye, R. C., "Composite Pd/Ta Metal Membranes for Hydrogen Separation," Journal of Membrane Science, 111, 123-133 (1996).

2. Peachey, N. M., Dye, R. C., Ries, P.D., Warren, M., and Olken, M., "Pulsed Laser Deposition of Zeolitic Thin Films: Novel Structures for Molecular Recognition," Journal of Porous Materials, 2, 331-339 (1996). 


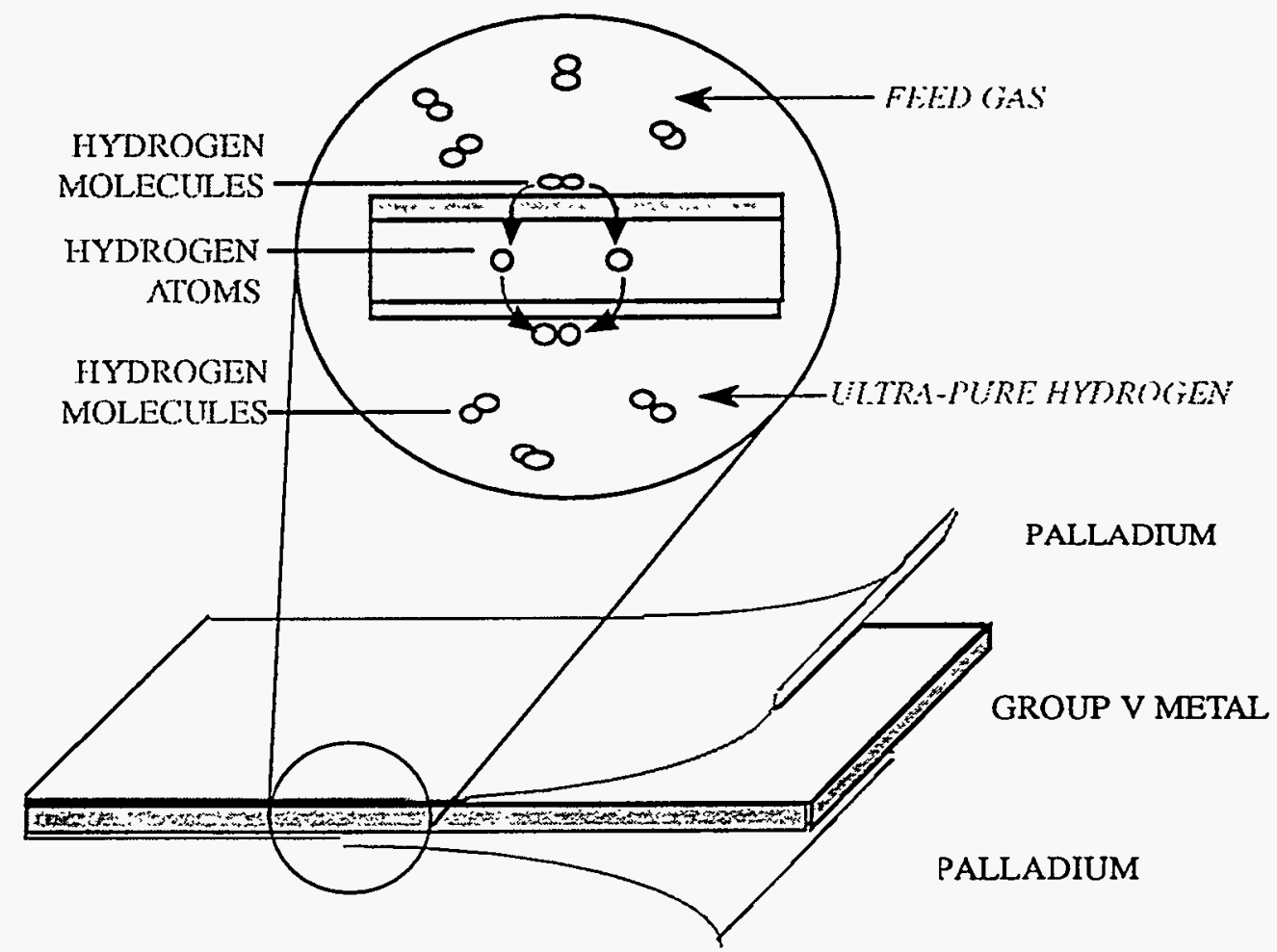

Figure 1. The multilayer metal membrane is composed of thin layers of palladium on the top and bottom of a Group V metal. 


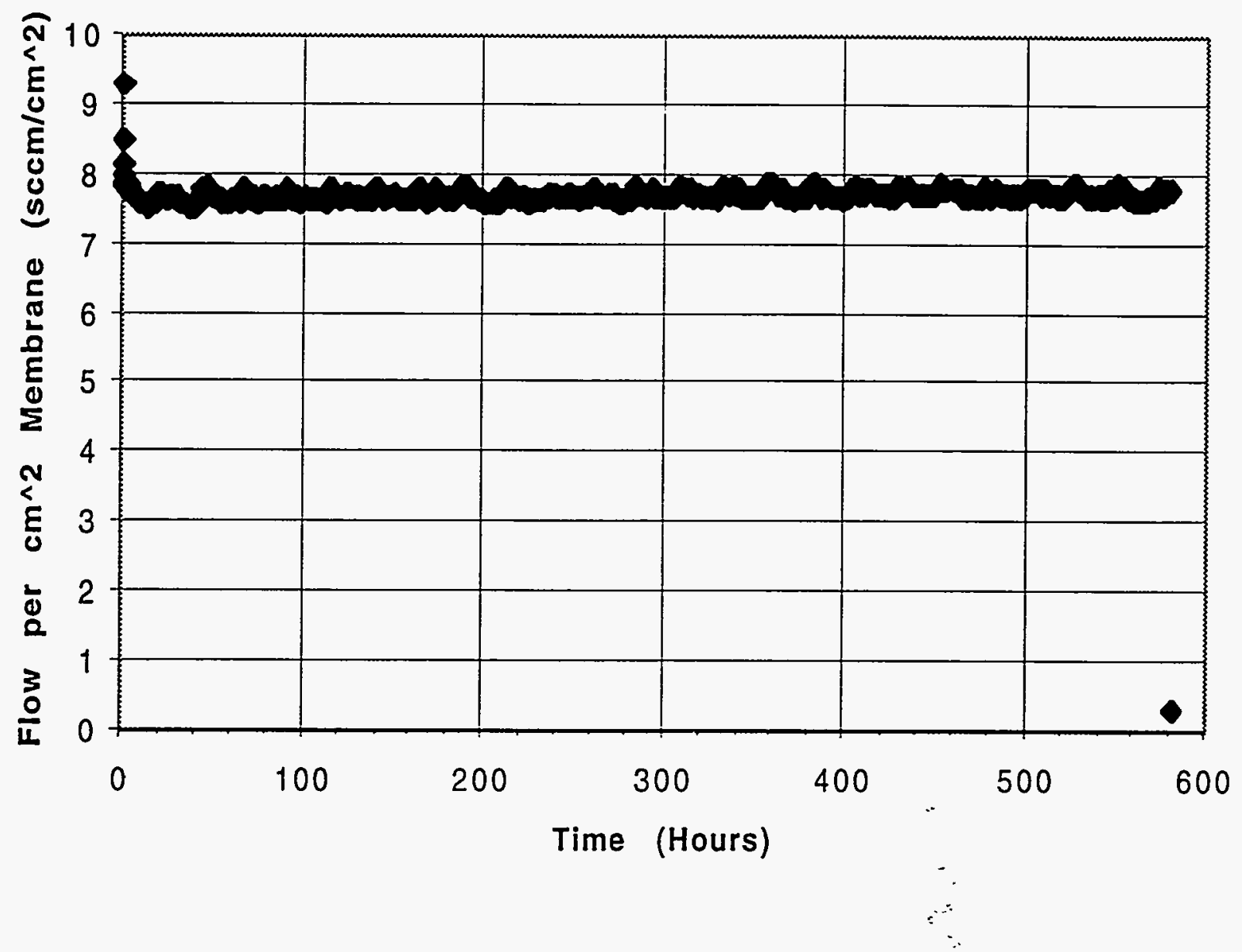

Figure 2. The hydrogen flow is stable at $7.78 \mathrm{sccm}$ per $\mathrm{cm}^{2}$ membrane and $84 \%$ efficiency in this $\mathbf{5 7 5}$ hour test. 